\title{
STUDY OF DEMOGRAPHIC AND CLINICAL PROFILE OF OCULAR TRAUMA
}

D. N. Prakash, K, Sathish, Abhijith Kumar, Anuradha Patil

1. Assistant Professor. Department of Ophthalmology, MMC \& RI, Mysore,

2. Associate Professor. Department of Ophthalmology, MMC \& RI, Mysore,

3. PG Student. Department of Ophthalmology, MMC \& RI, Mysore,

4. PG Student. Department of Ophthalmology, MMC \& RI, Mysore,

\section{CORRESPONDING AUTHOR:}

Dr. D. N. Prakash,

Assistant Professor, Dept. of Ophthalmology,

K. R. Hospital, Mysore Medical College and Research Institute,

Mysore- 570001.

E-mail: bhanup7@yahoo.com

Ph: 00919342184581.

ABSTRACT: OBJECTIVE: To analyze the demography and clinical profile of patients with ocular trauma. DESIGN: A prospective interventional study. PARTICIPANTS: Cases of ocular injuries who presented to our hospital between May 2010 and May 2011. RESULTS: A total of 90 cases were recorded, of whom $65.5 \%$ were male. A third of the injuries occurred in the age group of 25 to 40 years. Injury with a metallic object was the most common cause, accounting for $18.8 \%$ of the cases followed by wooden stick and stone. $72 \%$ of the cases presented to the hospital within 3 days of the injury, and most had poor visual acuity in the affected eye. Closed globe injuries were the most common diagnosis made at the presentation (50.01\%). Majority of the patients presented with visual impairment (61.1\%). CONCLUSION: Most ocular injuries in this study occurred at the workplace, suggesting the need to explore workplace strategies to minimize ocular trauma as a priority. Eye care programs may need to consider ocular trauma as a priority. Occupation specific ocular protection has to be developed and awareness regarding the same needs to be increased. A targeted approach focusing on productive ages and males may also help.

INTRODUCTION: Ocular trauma is an important, preventable public health problem worldwide. As many as half a million people in the world are blind as a result of ocular injuries. Trauma is most important cause of unilateral loss of vision particularly in developing countries accounting for $1.37 \%$ of overall blindness.(1)Ocular trauma has been an unavoidable hazard of industrialization in today's world.

Eyes comprise as little as $0.27 \%$ of the total body surface area and only $0.1 \%$ of the erect frontal profile. However, injuries to the eye are found in $10-13 \%$ of all combat casualties. One unfortunate thing about ocular trauma is that it affects primarily children and young persons (the productive age group) and secondly after severe trauma, prognosis remains quite guarded in spite of timely treatment.

The impact of trauma on a human eye may range from occurrence of minute corneal abrasions/innocuous sub-conjunctival hemorrhage to a badly lacerated globe. Few studies exist on the epidemiological profile of ocular injuries in South India. 
A prospective analysis of cases of ocular injuries presenting to our hospital during one year between May 2010 and May 2011 was done to study the type of injury, vision affected by injury, object causing the injury and activity being done at the time of injury. The findings were co-related with socio-demographic data.

MATERIALS AND METHODS: In this prospective interventional study conducted at department of Ophthalmology, K.R. Hospital, Mysore, 90 eyes of 90 patients with ocular injuries were studied. Patients presenting at our institute from May 2010 to May 2011 with diagnosis of ocular trauma were randomly included. Eyes with pre-existing ocular diseases and eyes with clinical findings suggestive of non-traumatic cause were excluded from the study.

The demographic data of each patient including address (rural/urban), literacy status, occupation, and financial status were recorded. Age and sex characteristics of the subjects were noted. History was noted regarding time since injury, relation with work and work place, object causing trauma, mode of injury (blunt/penetrating) and type of injury (open/ closed globe).

Visual acuity at the time of reporting and final visual acuity at the end of treatment were noted.The detailed ophthalmic workup of all the patients including slit lamp examination,+90D examination and indirect ophthalmoscopy was carried out. Ultrasonography was used whenever unclear media prevented fundus evaluation. Intraocular pressure was measured in all eyes except in fresh open globe injuries. Gonioscopy was done in all closed globe injuries. In eyes with corneal oedema and or hyphaemagonioscopy was done at next follow up.

Intraocular injuries were classified according to Birmingham Eye Trauma Terminology (BETT), which is as follows.(2)

Open globe injury was repaired at earliest possible time under local/ general anaesthesia. Closed globe injuries were initially managed medically and after evaluation necessary treatment was done. Posterior segment injuries were referred for vitreoretinal opinion and intervention.

All the patients were followed up at 1 week, 6 weeks and 3 months intervals. Patients referred for vitreo-retinal intervention were also followed up.

\section{RESULTS:}

1. The data from our study shows males are more prone to the injury than females. Male- $76.6 \%$ and female-23.3\%. male to female ratio was 3.28:1.Among males work related injury is more common where as in females housework related injuries were common.

2. Study data shows most of the injuries were work related $41.11 \%$ followed by play $30 \%$. Form work and laborers are more frequently prone to injuries.

3. The mean age group affected was $>40$ years followed by children $<15 y e a r s$.

4. According to study $68 \%$ injuries were blunt trauma and $32 \%$ penetrating injury.

5. There was wide variety of causative agents that resulted in eye injuries like metallic objects $18.8 \%$, wooden stick $15.55 \%$, stone $14.44 \%$.

DISCUSSION: The data from our study shows males are more prone to the injury than females. Male- $76.6 \%$ andfemale-23.3\%. Male to female ratio was 3.28:1. Among males work related injury is more common where as in females housework related injuries were common. 
Similar results were seen in previous study(3) done on epidemiology of hospitalised ocular injuries in the upper east region of GHANA done by GyasiME et al showed that males $(75 \%)$ are more affected than females for ocular injuries.

Our study data shows most of the injuries were work related $41.11 \%$ followed by play $30 \%$. Form work and labourers are more frequently proneto injury.

This is consistent with study done on "Epidemiology of Patients Hospitalized for Ocular Trauma in the Chaoshan Region of China, 2001-2010 "(1) which showed that the most frequent injury types among all cases were work-related injuries (46.5\%).(4)

Ocular trauma in a rural population of southern India(5): the Andhra pradesh eye disease study showed work related injuries $55.9 \%$.

Significant association B/W ocular injury and work place was found in Delhi eye study( ${ }^{(6)}$. In our study the mean age group affected was $>40$ years followed by children $<15 y e a r s$.

The percentage of children aged 14 years or less was 21.1 (95\% CI 18.9-23.4) during the study period based on the National Statistical Yearbook 2010.

According to our study $68 \%$ injuries were blunt trauma and $32 \%$ penetrating injury. Our study showed slightly more percentage of blunt injury than study done by Krishnaiah et al $54.9 \%$ blunt trauma in ocular trauma in south Indian population. ${ }^{(5)}$

Our study data shows there was wide variety of causative agents that resulted in eye injuries like metallic objects $18.8 \%$, wooden stick $15.55 \%$, stone $14.44 \%$.

Wooden stick was the most common object of the injury. It is noted by Dr.Deepa Abraham et al and Singh et al.

Most of the patients (60\%) had poor visual acuity at the time of presentation .with appropriate management the above figure drop down to $20 \%$ at the end of three months, which indicates if well treated, these cases of injury can have good recovery and also the importance of establishing the better access to medical facilities.

Gothwal VK et al (72.0\%) patients were blind at presentation. Post treatment (24.8\%) were blind. There was a $66.7 \%$ reduction in blindness and $54.1 \%$ reduction in visual impairment rates after treatment.(7)

In AIOC proceedings on trauma session 2008 study done by Mehul shah et al showed, $25.63 \%$ of the patients presented with visual acuity of $6 / 60$ or less and with appropriate management the above figure drop down to $17.32 \%{ }^{(8)}$

CONCLUSIONS: Most ocular injuries in this rural population occurred at the workplace, suggesting the need to explore workplace strategies to minimize ocular trauma as a priority. Eye care programs targeting high-risk ocular trauma groups may need to consider ocular trauma as a priority in eye health awareness strategies to reduce blindness due to trauma.

\section{REFERENCE:}

1. Dr.Madhulika Mandal, Dr.Sandeep Mitthal, Dr.Charu Mithal (Presenting Author: Dr.Madhulika Mandal)Evaluation of Various Prognostic Factors and Visual Outcome inOpen Globe Injuries in 51 Eyes of 50 Patients.2010 aioc proceedings.

2. Ramanjitasihota, Radhika Tandon. Injuries to the eye. In, Parsons' diseases of the eye, 21 st edition, Elsevier publication, 2011:375 
3. ME Gyasi, WMK Amoaku, and MA Adjuik. Epidemiology of Hospitalized Ocular Injuries in the Upper East Region of Ghana. Ghana Med J. 2007 December; 41(4): 171175.PMCID: PMC2350113.

4. He Cao, Liping Li, and Mingzhi Zhang. Epidemiology of Patients Hospitalized for Ocular Trauma in the Chaoshan Region of China, 2001-2010.

5. S Krishnaiah ,PK Nirmalan, BR Shamanna ,M Srinivas, GN Rao, Thomas R.Ocular trauma in a rural population of southern India: the Andhra Pradesh Eye Disease Study. Ophthalmology. 2006 Jul; 113(7):1159-64.

6. S Vats ,G V S Murthy ,M Chandra, S K Gupta, P Vashist and M Gogoi. Epidemiological study of ocular trauma in an urban slum population in Delhi, India. Indian J Ophthlmol.2008 Jul-Aug;56(4):313-316.

7. Gotwal V.K. et al. Demography and prognosticfactors of ocular injuries in South India. AUSTNZJ ophthalmology 1999;27:318-25.

8. Dr.Mehul Shah, Dr.AsitHasmukhlal Shah, Dr.Shreya Shah, Dr.AshitShah,Dr. SandipVora. Epidemiology of Ocular Trauma. 2010aiocproceedings.

\begin{tabular}{|r|r|r|r|r|r|r|}
\hline & LT 15 & 15 to 25 & 25 to 40 & GT 40 & Total & $\mathrm{N}$ \\
\hline Business & 0.000 & 0.000 & 2.222 & 3.333 & 5.556 & 5 \\
\hline farmer & 0.000 & 0.000 & 0.000 & 17.778 & 17.778 & 16 \\
\hline Housewife & 0.000 & 2.222 & 3.333 & 6.667 & 12.222 & 11 \\
\hline laboul & 0.000 & 5.556 & 16.667 & 1.111 & 23.333 & 21 \\
\hline mechanic & 0.000 & 1.111 & 1.111 & 1.111 & 3.333 & 3 \\
\hline office worker & 0.000 & 0.000 & 0.000 & 1.111 & 1.111 & 1 \\
\hline student & 26.667 & 10.000 & 0.000 & 0.000 & 36.667 & 33 \\
\hline Total & 26.667 & 18.889 & 23.333 & 31.111 & 100.000 & \\
\hline $\mathrm{N}$ & 24 & 17 & 21 & 28 & & \\
\hline
\end{tabular}

\begin{tabular}{|r|r|r|r|}
\hline Test statistic & Value & df & Prob \\
\hline Pearson Chi-square & $896^{124}$ & $0{ }^{18.0}$ & $0^{0.00}$ \\
\hline Likelihoodratio Chi-square & $199{ }^{142 .}$ & $00^{18.0}$ & $0^{0.00}$ \\
\hline
\end{tabular}

\begin{tabular}{|r|r|}
\hline Coefficient & Value \\
\hline Contingency & $62^{0.7}$ \\
\hline
\end{tabular}


Fig. 1. BETTS. The double-framed boxes show the diagnoses that are used in clinical practice

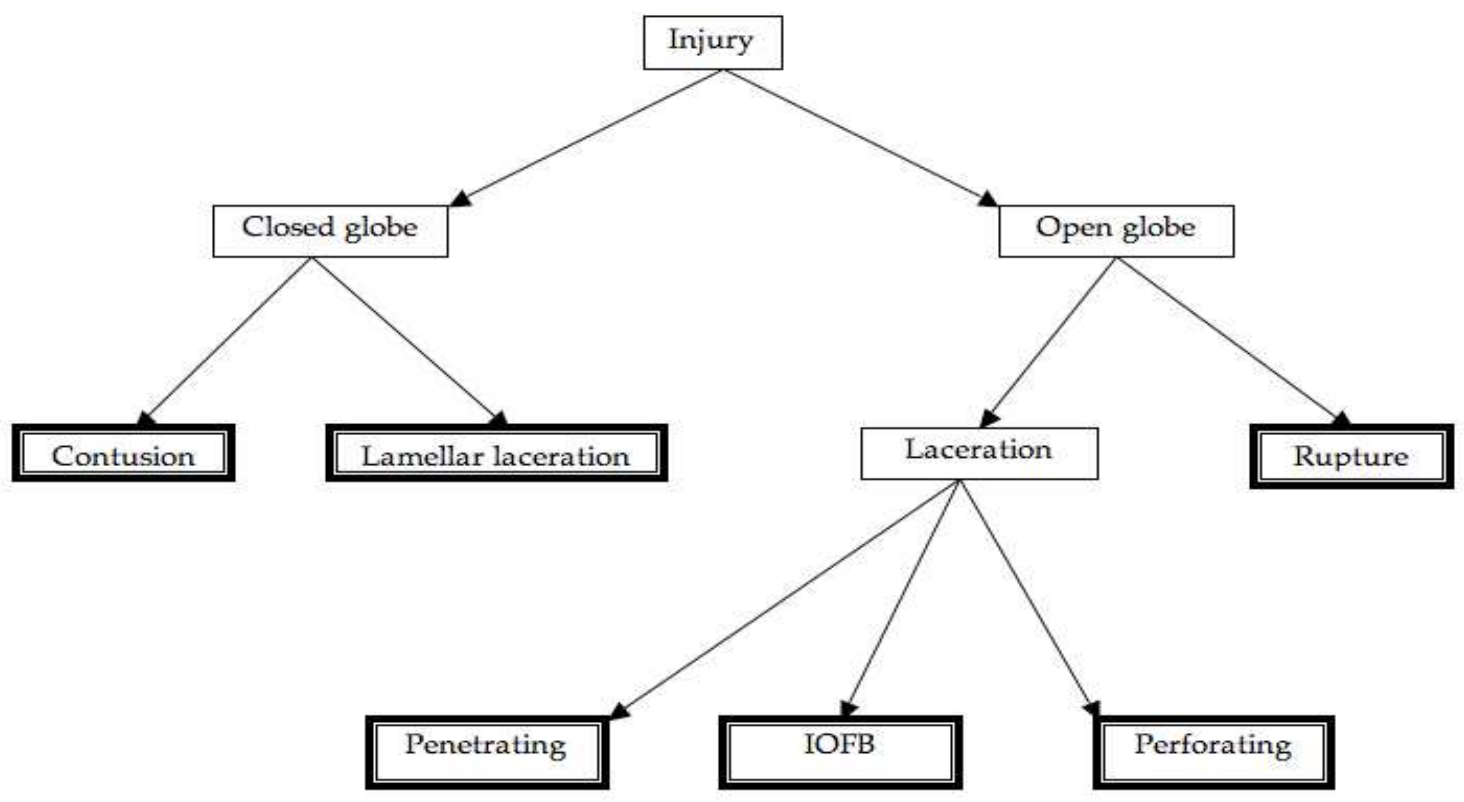

Distribution of Gender

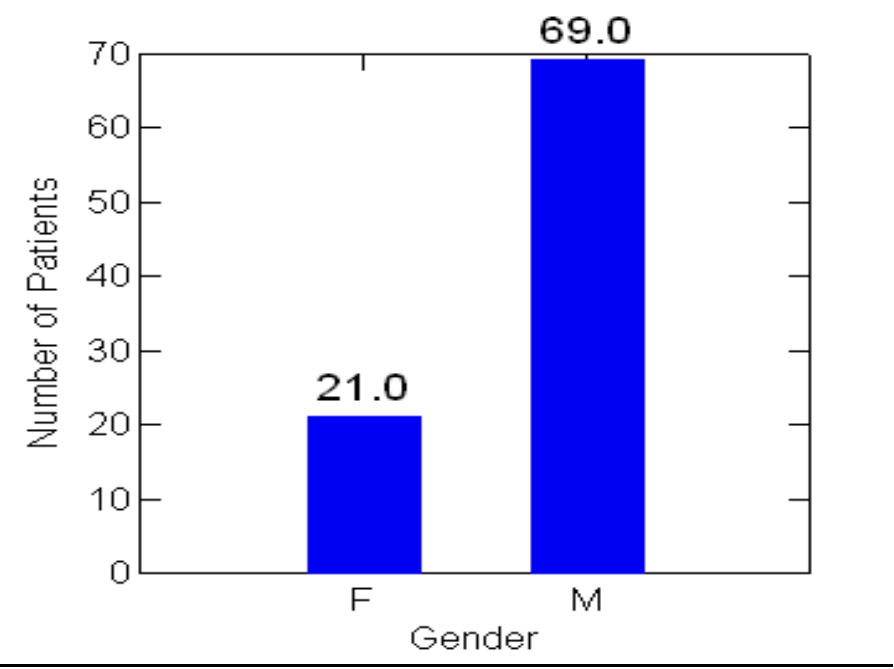




\section{Distribution of Age and Gender}

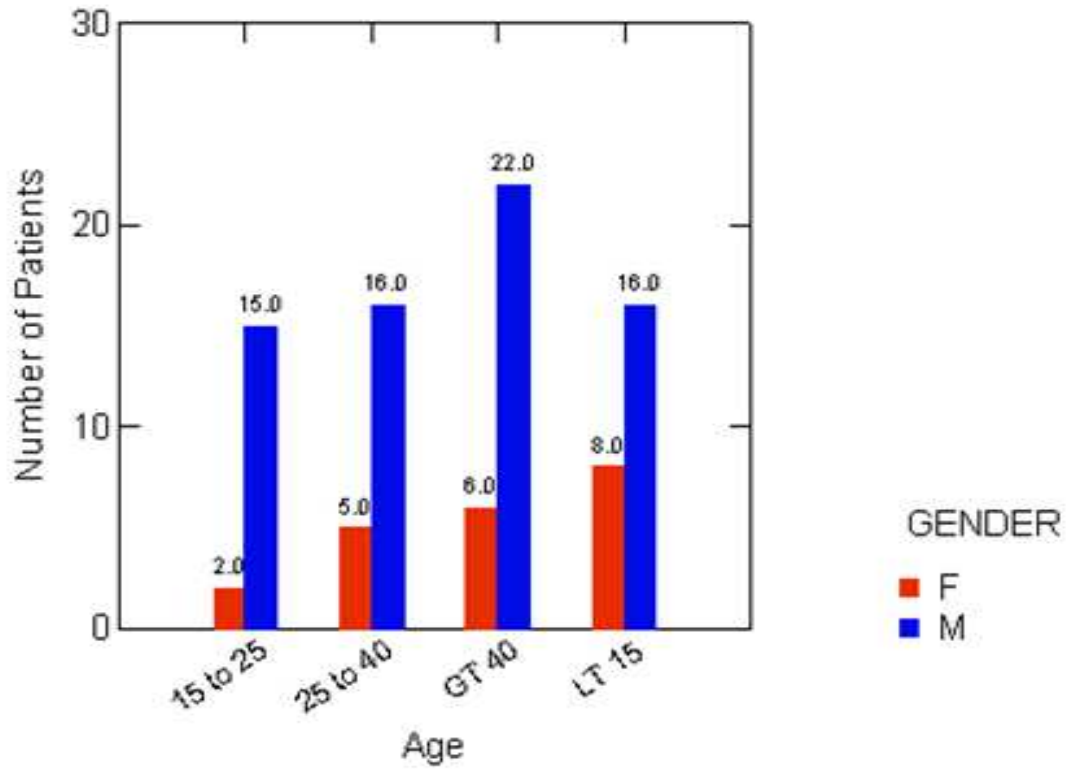

P-value Table for Significance test and Contingency Coefficient values

\begin{tabular}{lrrr}
\hline Significance Between & Chi-square & $\begin{array}{l}\text { Likelihood Chi- } \\
\text { square }\end{array}$ & \multicolumn{2}{c}{$\begin{array}{l}\text { Contingency } \\
\text { Coefficient }\end{array}$} \\
\hline Gender VS Age & $\mathbf{0 . 4 4 5}$ & $\mathbf{0 . 4 2 8}$ & 0.17 \\
Occupation VS Gender & 0 & 0 & 0.538 \\
Injury Causes VS Gender & $\mathbf{0 . 8 8 4}$ & $\mathbf{0 . 7 8 7}$ & 0.287 \\
Activity VS Gender & 0.002 & 0.001 & 0.463 \\
Type VS Gender & $\mathbf{0 . 7 1 5}$ & $\mathbf{0 . 7 1 5}$ & 0.039 \\
Specific Ini VS Gender & $\mathbf{0 . 7 9 7}$ & $\mathbf{0 . 6 3 8}$ & 0.106 \\
Coniuctival VS Gender & $\mathbf{0 . 3 5 6}$ & $\mathbf{0 . 2 2 3}$ & 0.37 \\
Occupation VS Age & 0 & 0 & 0.762 \\
Sight at present VS One month & 0 & 0 & 0.753 \\
Sight at present VS Three & 0 & & 0.832 \\
month & & & 0 \\
\hline
\end{tabular}

